\title{
Antiobesity activity of Zingiber officinale
}

\author{
Iram Nazish ${ }^{* 1}$, S H Ansari², Poonam Arora², Adil Ahmad² \\ 'Department of Pharmacology, HK College of Pharmacy, Oshiwara, Mumbai, INDIA. \\ 2Department of Pharmacognosy and Phytochemistry, Faculty of Pharmacy, Hamdard University, Hamdard Nagar, New Delhi, INDIA.
}

\begin{abstract}
Context: Zingiber officinale Roscoe (Zingiberaceae) rhizome, known commonly as ginger is extensively used in Indian traditional system of medicine for treatment of various disorders. The ethanolic Z. officinale extract is reported to have various activity such as antidiabetic, antihyperlipidemic and antioxidant activity in experimental animals. Objective: To evaluate anti-obesity effect of aqueous $Z$. officinale extract in murine model of high fat diet (HFD)- induced obesity. Materials and Methods: Male Wistar rats fed with HFD (20 g/day/rat, p.o) for a period of 42 days were used to induce obesity. Aqueous Z. officinale extract $(20 \mathrm{mg} / \mathrm{kg} \mathrm{b.w}$.) administered orally to HFD fed rats from day 8 to 50 days for a period of 42 days. Body weight gain, serum lipids, insulin and leptin parameters were measured. Results: Oral feeding of the aqueous $Z$. officinale extract $(20 \mathrm{mg} / \mathrm{kg}$ ) to HFD-induced obese rats for a period of 42 days resulted in
\end{abstract}

significant reduction in body weight gain, insulin, leptin, lipids as compared to rats fed HFD alone. Further, the extract also showed significant increase in high density lipoprotein (HDL-C) levels. Discussion and Conclusion: These results show that aqueous $Z$. officinale extract possess significant anti-obesity potential.

Key words: Zingiber officinale, Leptin, High-fat diet, Rat, Insulin.

Corresponding author: Iram Nazish, Department of Pharmacology, HK College of Pharmacy, Oshiwara, Mumbai, INDIA.

Phone: +917506375184

Email: iramnazishpharma@gmail.com

DOI : $10.5530 /$ pj.2016.5.5

\section{INTRODUCTION}

Obesity generally described as a condition of abnormal body weight resulting from an accumulation of extra adipose tissue, basically in response to a state of positive energy balance that occurs when intake exceeds energy expenditure. It is a growing global health problem in the present era. Obesity is a chronic relapsing, stigmatized neurochemical disease that is more prevalent in developing and developed countries and leading to much comorbidities. ${ }^{1}$

Obesity, which is associated with serious moralities including a high incidence of type 2 diabetes, hyperlipidemia, hypercholestrolemia, cardiovascular disease, osteoarthritis, and many more diseases. ${ }^{1-2}$ Obesity is a disorder not a disease, but, it has been recognized as a chronic disease by National Institute of health consensus conference in 1985 due to the increasing prevalence and associated risks. ${ }^{2}$ This situation currently prevailing widely, anti-obesity drugs expanding rapidly in the market, but drugs are sparse in relation to need. Hence, novel anti-obesity drug is required to handle the problem.

Plants and natural products are long been recognized as an important sources for the therapeutically effective medicines. Approximately $80 \%$ of the world's population relies on plants for the natural products and medication. Of the 520 new drugs approved between 1983 and 1994, $39 \%$ were of natural origin and $60-80 \%$ of antibacterial and anticancer drugs were derived from natural products. Infect several Indian medicinal plants or extracts have been used as anti-obesity plant. ${ }^{3}$

Z. officinale, commonly known as Ginger, (family Zingiberaceae). Z. officinale rhizome contains $1-4 \%$ essential oil and an oleoresin. The essential oil varies as a function of geographical origin, but the chief constituent is sesquiterpene hydrocarbons. These compounds include (-)-zingiberene, (+)-ar-curcumene, (-)- $\beta$ - sesquiphellandrene, and $\beta$-bisabolene. The rhizome cure tumors, ascites, bronchitis, jaundice and mental disorders, also have antidiabetic, antidyslipidemic, cardioprotective and antioxidant. ${ }^{4-6}$

However, aqueous extract of $Z$. officinale has not been investigated for its anti-obesity potential so far. Hence, this study was carried out to test the hypothesis that aqueous extract of $Z$. officinale may have anti-obesity activity in high fat diet (HFD)- induced obesity in Wistar rats.

\section{MATERIALS AND METHODS}

\section{Plant material}

Dried Z. officinale fruits were purchased from Local market Kharibaoli, Delhi and authenticated by Dr. H. B. Singh, expert taxonomist, National Institute of Science Communication and Information Resources (NISCAIR), New Delhi, India.

\section{Preparation of aqueous Z. officinale extract}

All plant materials were washed; shade dried and powdered, then extracted with water in a soxhlet apparatus for 72 hours. The solvent was removed under reduced pressure to give a dry extract, $5 \%$ yield $\mathrm{w} / \mathrm{w}$ with respect to the crude material and stored at $-20^{\circ} \mathrm{C}$ till experiment was not started. The weighed amount of aqueous extract was suspended in $1 \%$ gum acacia in normal saline for pharmacological activity. This extract was used for phytochemical analysis for standardization.

\section{Macroscopical studies}

The material of $Z$. officinale were subjected to macroscopical studies which comprised of organoleptic characteristics of the drug viz., size, colour, odour, taste, smell, texture, fracture etc.

\section{Microscopical studies}

Qualitative microscopic evaluation was carried out by taking powder. $5 \mathrm{~g}$ of the drug sample was taken, powdered. The powdered material was taken on an 85 mesh sieve and allowed in slow running water for washing away the minerals. The materials were cleared in chloral hydrate, wash with distilled water and mounted in glycerin, then observed characters. ${ }^{7,8}$

\section{Animals and Diets}

The female Wistar rats (100-150 g) were procured from central animal house facility of Hamdard University, New Delhi, India. The animals 
were housed in standard polypropylene cages and maintained under controlled room temperature $\left(22 \pm 2^{\circ} \mathrm{C}\right)$ and humidity $(55 \pm 5 \%)$ with $12 \mathrm{~h}$ light and $12 \mathrm{~h}$ dark cycle. All the rats in Normal group were provided with commercially available rodent chow diet (Amrut rat feed, Nav Maharastra Chakan Oil Mills Ltd, Delhi, India) and tap water ad libitum. The guidelines of committee for the purpose of control and supervision of experiments on animals (CPCSEA), Government of India were followed and prior permission was sought from the Institutional Animal Ethics Committee, Jamia Hamdard, New Delhi for conducting the study.

\section{HFD- induced obesity}

Rats were fed a HFD (20 g/rat/day) in a pellet form and water ad libitum for the period of 6 weeks. HFD was purchased from National Centre for Laboratory Animal Science (NCLAS), National Institute of Nutrition (NIN), Hyderabad, India. Composition of HFD is shown in (Table 1).

\section{Experimental Design}

In this study, a total of 50 rats were used and divided into five groups of 6 rats each. Group 0020I: normal healthy control rats fed with rodent chow diet and administered $1 \%$ gum acacia ( $1 \mathrm{ml} / \mathrm{kg}$, body weight (bw)) for the period of 42 days (NC); Group II: obesity control rats fed with HFD (20g/rat/day) for the period of 42 days (HFD); Group III: rats fed with HFD (20g/rat/day) for the period of 42 days, treated with aqueous Z. officinale extract $(20 \mathrm{mg} / \mathrm{kg}$, bw) for period of 42 days $(\mathrm{HFD}+\mathrm{ZO})$; Group IV: rats fed with HFD (20g/rat/day) for the period of 42 days, treated with Orlistate ( $5 \mathrm{mg} / \mathrm{kg}$, bw) for period of 42 days (HFD+ ORL); Group V: rats fed with rodent chow diet for the period of 42 days + treated with aqueous $Z$. officinale extract $(20 \mathrm{mg} / \mathrm{kg}$, bw) for period of 42 days (ZO per se). All the drugs were administered orally via a standard orogastric cannula (Table 2).

Food and water intakes were measured daily for the period of 42 days at the same time. Food intake and water intake were measured on per cage basis ( 6 rats per cage) and the average food and water consumed were calculated. At the end of the experimental period (on 43 th day), the animals were anesthetized with ether, following overnight fasting. Blood was drawn by retro-orbital method into a tube and the serum was obtained by centrifugation. After collection of blood, rats were sacrificed; heart and perirenal, epididymal, mesenteric fat pads were excised immediately, rinsed with phosphate buffer saline and weighed. The serum and heart samples were stored at $-70^{\circ} \mathrm{C}$ until analysis.

\section{Anthropometric measurements}

The body weights were determined once a week. Body mass index (BMI) was calculated from formula:

$$
\mathrm{BMI}=\text { Body weight }(\mathrm{g}) / \text { length }{ }^{2}\left(\mathrm{~cm}^{2}\right)
$$

\section{Biochemical serum analyses}

Serum glucose, triglyceride (TG) and total cholesterol (TC) levels were determined by enzymatic methods using commercial assay kits (SPAN Diagnostics Ltd, Surat, India) according to the manufacturer's protocols. Serum High-density lipoprotein cholesterol (HDL- C) levels were determined using HDL Cholesterol Test Kit (Reckon Diagnostics P. Ltd, Baroda, India). Serum leptin and insulin levels were measured by immunoassays using a commercially available ELISA assay kit from Ray Biotech, Inc (Norcrcoss, US) and Crystal Chem Inc (Downers Grove, IL 60515, USA) respectively. Low-density lipoprotein cholesterol (LDL-C) levels were calculated using Friedewald formula. ${ }^{9}$ Serum very low density lipoprotein cholesterol fraction (VLDL-C) concentration was calculated by deduction of the sum of HDL-C and LDL-C concentrations from that of TC.

\section{Statistical analysis}

Data are expressed as the mean \pm standard error of the mean (SEM). The statistical significance of difference between the mean values for the treatment groups was analysed by ANOVA (analysis of variance) followed by Dunnett's t- test using Graph pad InStat ${ }^{\circledR}$ version 3.06 (Graph Pad Software, San Diego, CA, USA). Values of $\mathrm{p}<0.05$ were considered significant.

\section{RESULTS}

\section{Macroscopical Studies}

The Z. officinale are light brown colored irregular shaped rhizome, odour pleasant, Pungent taste and rough textured shown in (Figure.1). The organoleptic evaluations of $Z$. officinale were showed in (Table 3).

\section{Microscopic study}

For microscopic study, $5 \mathrm{~g}$ of the drug sample was taken, powdered. The powdered material was

taken on a 85 mesh sieve and allowed in slow running water for washing away the minerals. The materials were cleared in chloral hydrate, wash with distilled water and mounted in glycerin, then observed charcters ${ }^{8}$ (Figure 2).

\section{Effect on Food and Water intake}

Negligible $(\mathrm{P}>0.05)$ change in food and water intake was observed in HFD fed group as compared to the NC group. Administration of aqueous $Z$. officinale extract $(20 \mathrm{mg} / \mathrm{kg}$ ) along with HFD (HFD + ZO group) did not produce significant change in the food and water intake as compared to HFD group (Table 4).

\section{Effect on anthropometric Parameters}

In the HFD group body weight gain of rats was greater than the values for the NC. HFD supplemented with $20 \mathrm{mg} / \mathrm{kg}$ aqueous $Z$. officinale extract reduced body weight gain of the rats. However, there was no significant difference in body weight gain among the $\mathrm{NC}$ and $\mathrm{ZO}$ per se treated groups (Table 4).

\section{Effect on organ weight and fat pad analysis}

No significant changes in weights of heart, kidney, liver and spleen in drug treated albino rats as compared to control and high fat diet fed group except weight of kidney was reduced significantly $(\mathrm{P}<0.05)$. However, drug treatment has variably restricted increase in fat pad weights compared to high fat diet group significantly $(\mathrm{P}<0.01)$ (Table $5 \& 6$ _).

\section{Effect on serum lipid levels}

Serum TG levels were significantly higher in HFD fed group compared to that of NC group, HFD+ ZO group $(p<0.01)$. Levels of serum TC, LDL-C, VLDL-C were similar to tendency of serum triglyceride level $(\mathrm{p}<0.01)$. However, TC, LDL-C and HDL-C were significantly $(\mathrm{p}<0.05)$ affected in HFD + ZO treated group Serum levels of HDL-C were higher in HFD+ ORL treated group compared to HFD fed and NC group, significant difference was recognized $(\mathrm{p}<0.001)$ (Table 7$)$. 
Table 1: Compositions of the HFD (g/kg diet)

\begin{tabular}{cc}
\hline Ingredients & Quantity \\
\hline Casein & 342.0 \\
L-Cystine & 3.0 \\
Starch & 172.0 \\
Sucrose & 172.0 \\
Cellulose & 50.0 \\
Ground nut oil & 25.0 \\
Tallow & 190.0 \\
AIN Salt mix & 35.0 \\
AIN Vitamin mix & 10.0 \\
Total (g) & 999.0 \\
\hline
\end{tabular}

Table 2: Experimental design

\begin{tabular}{cc}
\hline Groups & No. of animals \\
\hline Normal control & 6 \\
Obese control (HFD) & 6 \\
HFD + Z. officinale $(20 \mathrm{mg} / \mathrm{kg})$ & 6 \\
HFD + Orlistat std $(5 \mathrm{mg} / \mathrm{kg})$ & 6 \\
Z. officinale per se & 6 \\
& 30 \\
\hline
\end{tabular}

Table 3: Organoleptic properties of Z. officinale

\begin{tabular}{cc}
\hline Colour & Light brown \\
\hline Shape & Irregular \\
Odour & Pleasant \\
Texture & Rough \\
Taste & Pungent \\
\hline
\end{tabular}

Table 4: Effect of aqueous $Z$. officinale extract $(20 \mathrm{mg} / \mathrm{kg}$ ) on food intake, water intake, and body weight gain in HFD-induced obesity in wistar rats

\begin{tabular}{cccc}
\hline Groups & $\begin{array}{c}\text { Food intake (gm/ } \\
\text { day/rat) }\end{array}$ & $\begin{array}{c}\text { Water intake } \\
\text { (ml/day/rat) }\end{array}$ & $\begin{array}{c}\text { Body weight } \\
\text { gain }(\mathbf{g})\end{array}$ \\
\hline NC & $14.91 \pm 0.26$ & $38.34 \pm 4.81$ & $74.67 \pm 1.26$ \\
HFD & $20.87 \pm 0.35$ & $41.6 \pm 8.87$ & $125.33 \pm 1.27$ \\
HFD+ZO & $19.15 \pm 0.26$ & $38.5 \pm 1.98 \mathrm{~ns}$ & $112.67 \pm 1.33$ \\
HFD+ ORL & $18.16 \pm 0.39 \mathrm{~ns}$ & $37.8 \pm 1.91 \mathrm{~ns}$ & $91.50 \pm 1.34^{* *}$ \\
ZO per s & $17.89 \pm 0.54$ & $40.8 \pm 2.38$ & $103.8 \pm 1.59$ \\
\hline
\end{tabular}

Data are expressed as Mean \pm SD $(n=6)$. Statistical significance in comparison to High Fat Diet

group at ${ }^{*} \mathrm{P}<0.05,{ }^{* *} \mathrm{P}<0.01,{ }^{* * *} \mathrm{P}<0.001$. One way ANOVA followed by Dunnet's t-test.

\section{Effect on serum insulin, lipase and leptin levels}

It was observed after 42 days of treatment, HFD + ZO group showed serum lipase levels in HFD fed group was significantly higher than that of normal control but significant reduction observed in HFD $+\mathrm{ZO}$ group $(\mathrm{p}<0.05)$. Result of HFD + ORL group, HFD + ZO group were mentioned in Table 8.

\section{Histopathological studies}

Results reveal that drug significantly reduced the fat accumulation in liver, heat and kidney (Figure 3, 4 \& 5_respectively).
Table 5: Effect of aqueous Z. officinale extract $(20 \mathrm{mg} / \mathrm{kg})$ on organ weight profile in albino rats after $\mathbf{4 2}$ days of treatment

\begin{tabular}{ccccc}
\hline \multicolumn{5}{c}{ Organ weight $(\mathrm{g} / 100 \mathrm{~g}$ of animal weight) } \\
\hline Groups & Heart & Kidney & Liver & Spleen \\
\hline NC & $0.607 \pm 0.091$ & $1.020 \pm 0.240$ & $6.981 \pm 0.580$ & $0.606 \pm 0.041$ \\
HFD & $0.838 \pm 0.034$ & $1.493 \pm .291$ & $9.376 \pm 0.195$ & $0.805 \pm 0.027$ \\
HFD+ZO & $0.782 \pm 0.084$ & $1.214 \pm 0.305^{*}$ & $7.809 \pm 0.767$ & $0.698 \pm 0.097$ \\
HFD+ORL & $0.725 \pm 0.055^{*}$ & $1.088 \pm 0.157^{* *}$ & $7.171 \pm 0.976^{* *}$ & $0.643 \pm 0.062$ \\
ZO per se & $0.659 \pm 0.54$ & $1.179 \pm 0.38$ & $7.685 \pm 0.59$ & $0.654 \pm 0.77$ \\
\hline
\end{tabular}

Data are expressed as Mean \pm SD $(n=6)$. Statistical significance in comparison to High Fat Diet

group at ${ }^{\star} \mathrm{P}<0.05,{ }^{* *} \mathrm{P}<0.01,{ }^{* *} \mathrm{P}<0.001$. One way ANOVA followed by Dunnet's t-test.

Table 6: Effect of aqueous Z. officinale extract $(20 \mathrm{mg} / \mathrm{kg}$ ) on Fat pad weight profile in albino rats after $\mathbf{4 2}$ days of treatment

\begin{tabular}{cccc}
\hline & \multicolumn{3}{c}{ Fat pad weights $(\mathrm{g} / 100 \mathrm{~g}$ of animal weight) } \\
\hline Groups & Mesentric & Perirenal & Uterine \\
\hline NC & $0.462 \pm 0.076$ & $0.74 \pm 0.032$ & $0.725 \pm 0.070$ \\
HFD & $0.931 \pm 0.086$ & $1.22 \pm 0.288$ & $1.035 \pm 0.118$ \\
HFD+ZO & $0.563 \pm 0.080^{* *}$ & $0.84 \pm 0.092^{* *}$ & $0.809 \pm 0.104$ \\
HFD+ORL & $0.511 \pm 0.086^{* * *}$ & $0.78 \pm 0.084^{* * *}$ & $0.754 \pm 0.092^{*}$ \\
ZO per se & $0.559 \pm 0.56$ & $0.81 \pm 0.14$ & $0.792 \pm 0.71$ \\
\hline
\end{tabular}

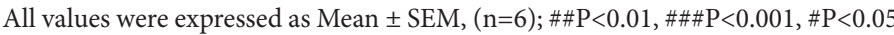
as compared to the NC group; one way ANOVA followed by Dunnet's t-test.

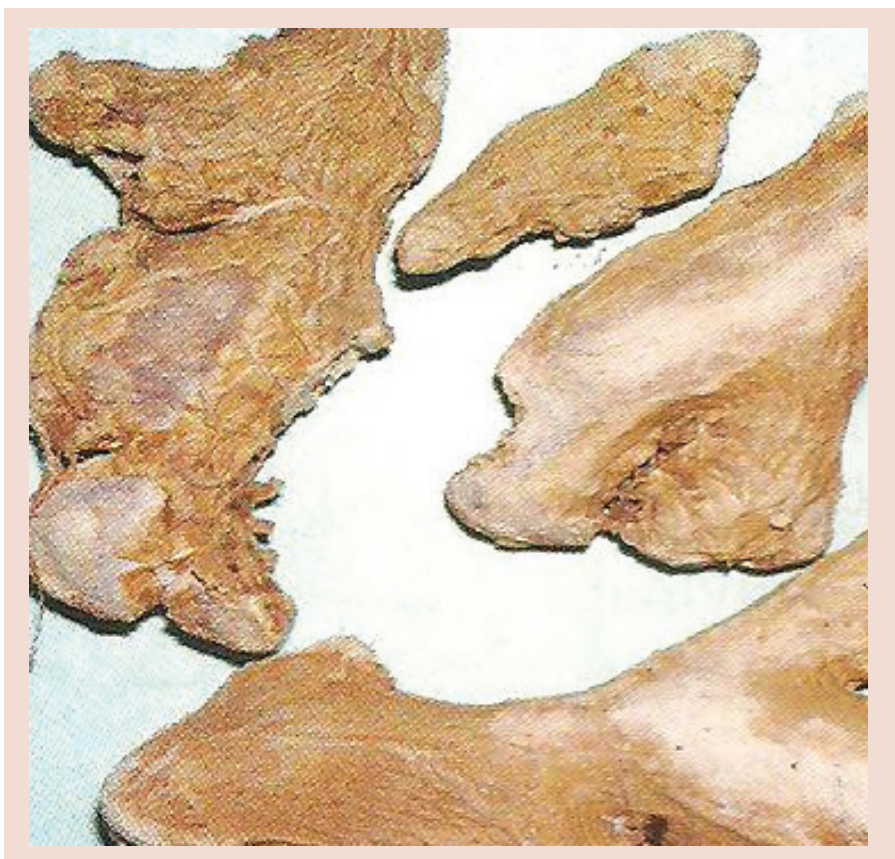

Figure 1: Dried rhizome of Zingiber officinale. 
Table 7: Effect of aqueous Z. officinale extract $(20 \mathrm{mg} / \mathrm{kg}$ ) on serum TC, TG, LDL-C, VLDL-C and HDL-C levels in HFD-induced obesity in wistar rats

\begin{tabular}{cccccc}
\hline Groups & $\mathrm{TC}(\mathrm{mg} / \mathrm{dl})$ & $\mathrm{TG}(\mathrm{mg} / \mathrm{dl})$ & $\mathrm{LDL}-\mathrm{C}(\mathrm{mg} / \mathrm{dl})$ & $\mathrm{VLDL}-\mathrm{C}(\mathrm{mg} / \mathrm{dl})$ & $\mathrm{HDL}-\mathrm{C}(\mathrm{mg} / \mathrm{dl})$ \\
\hline $\mathrm{NC}$ & $87.43 \pm 3.40$ & $69.44 \pm 12.21$ & $40.03 \pm 2.26$ & $13.89 \pm 2.44$ & $33.51 \pm 6.95$ \\
$\mathrm{HFD}$ & $164.76 \pm 24.26$ & $171.30 \pm 25.17$ & $107.28 \pm 9.54$ & $34.26 \pm 5.03$ & $23.23 \pm 9.69$ \\
$\mathrm{HFD}+\mathrm{ZO}$ & $146.15 \pm 21.39^{*}$ & $149.07 \pm 34.19$ & $84.35 \pm 6.91^{*}$ & $29.81 \pm 6.84$ & $31.99 \pm 7.65^{*}$ \\
$\mathrm{HFD}+\mathrm{ORL}$ & $114.14 \pm 13.20^{* * *}$ & $110.19 \pm 20.04^{* * *}$ & $53.81 \pm 2.58^{* * *}$ & $22.04 \pm 4.01^{* *}$ & $38.30 \pm 9.44^{* *}$ \\
ZO per se & $121.91 \pm 0.67$ & $132.05 \pm 1.01$ & $78.18 \pm 7.01$ & $26.10 \pm 0.20$ & $29.66 \pm 0.57$ \\
\hline
\end{tabular}

Data are expressed as Mean \pm SD $(n=6)$. Statistical significance in comparison to High Fat Diet group at ${ }^{*} \mathrm{P}<0.05$, ${ }^{* *} \mathrm{P}<0.01,{ }^{* * *} \mathrm{P}<0.001$. One way ANOVA followed by Dunnet's t-test.

Table 8: Effect of aqueous Z. officinale extract $(20 \mathrm{mg} / \mathrm{kg})$ on serum Insulin and leptin levels in HFD-induced obesity in wistar rats

\begin{tabular}{cccc}
\hline Groups & Insulin $(\mathrm{ng} / \mathrm{ml})$ & Leptin $(\mathrm{ng} / \mathrm{ml})$ & Lipase $(\mathrm{U} / \mathrm{L})$ \\
\hline NC & $0.91 \pm 0.21$ & $5.91 \pm 0.96$ & $661.32 \pm 54.12$ \\
HFD & $1.81 \pm 0.21$ & $19.77 \pm 2.34$ & $1109.94 \pm 218.23$ \\
HFD + ZO & $1.56 \pm 0.39$ & $17.82 \pm 1.93$ & $943.67 \pm 102.19^{*}$ \\
HFD + ORL & $1.12 \pm 0.16^{* * *}$ & $12.58 \pm 1.59^{* * *}$ & $861.11 \pm 102.89^{* * *}$ \\
ZO per se & $1.19 \pm 0.67$ & $16.05 \pm 1.01$ & $921.12 \pm 11.2$ \\
\hline
\end{tabular}

Data are expressed as Mean $\pm S D(n=6)$. Statistical significance in comparison to High Fat Diet group at ${ }^{*} \mathrm{P}<0.05,{ }^{* *} \mathrm{P}<0.01,{ }^{* *} \mathrm{P}<0.001$. One way ANOVA followed by Dunnet's t-test.

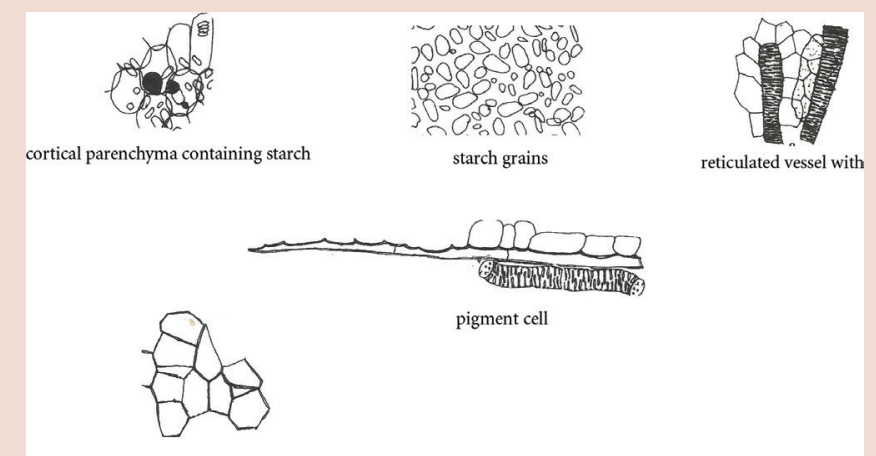

fibre fragment with attached reticulated vessel

surface view of cork

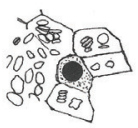

cortical parenchyma embedded with starch grains and oleoresin cell

Figure 2: Microscopical characters of $Z$. officinale.

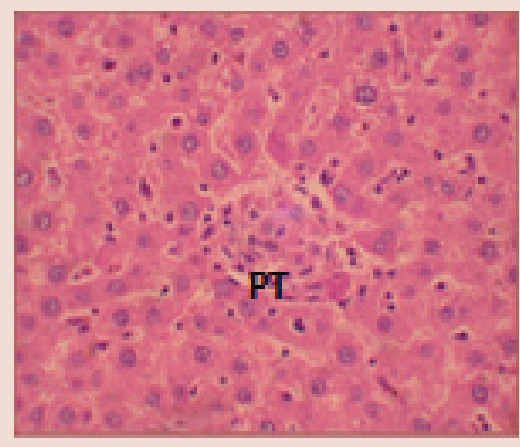

High power photomicrograph of liver from $\mathrm{ND}^{*}$ control group showing a normal portal triad (PT) and hepatocytes (H\&E x 400).

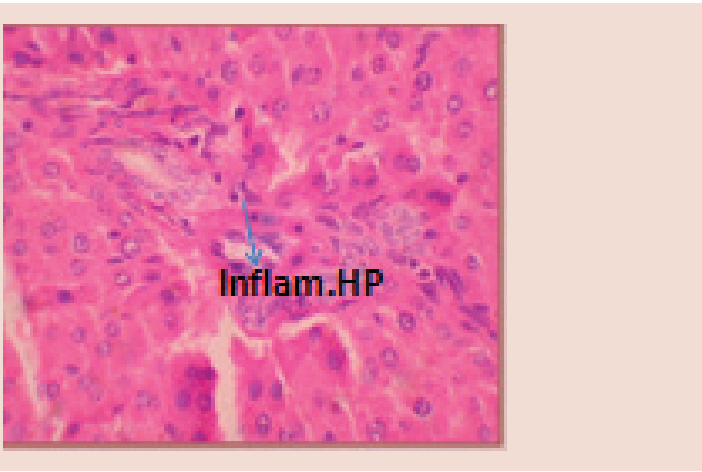

High power photomicrograph of liver from $\mathrm{HFD}^{\star}$ control group showing swelling of hepatocytes (HP) and narrowing sinusoids (H\&E x 400).

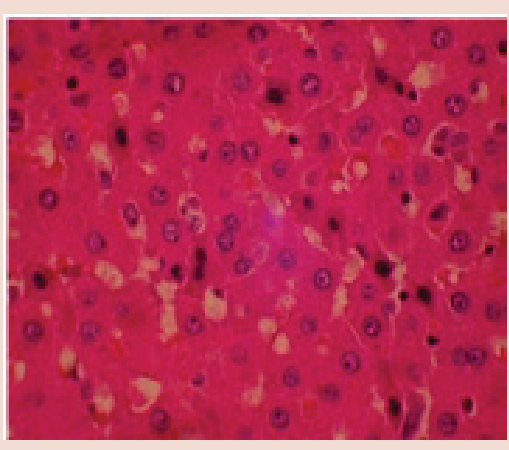

High power photomicrograph of liver from Z. officinale treated group showing a lot of fatty change (H\&E x 400).

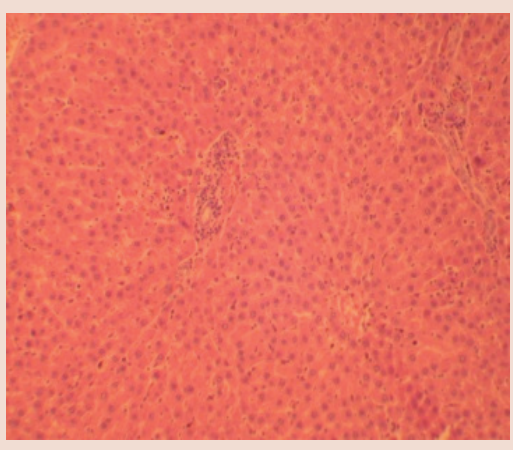

High power photomicrograph of liver from standard group showing no fat deposition (H\&E x 400). 


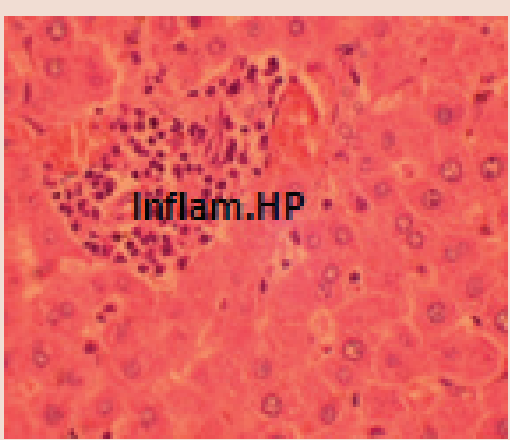

High power photomicrograph of liver from per se group showing significant inflammation of hepatocytes and damage to liver (H\&E x 400).

Figure 3: Changes observed in liver of different groups.

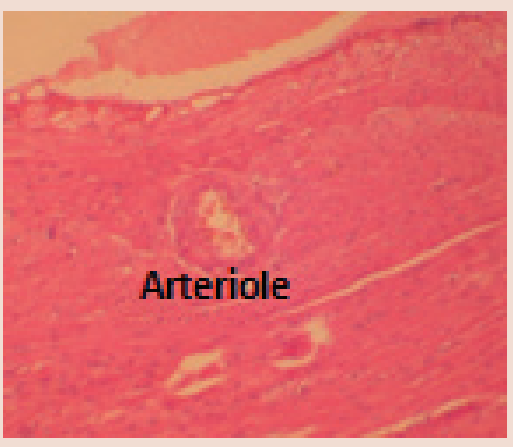

High power photomicrograph of Heart from $\mathrm{ND}^{*}$ control group showing normal arteriole (A) and muscles (M-myofibrils) (H\&E x 400).

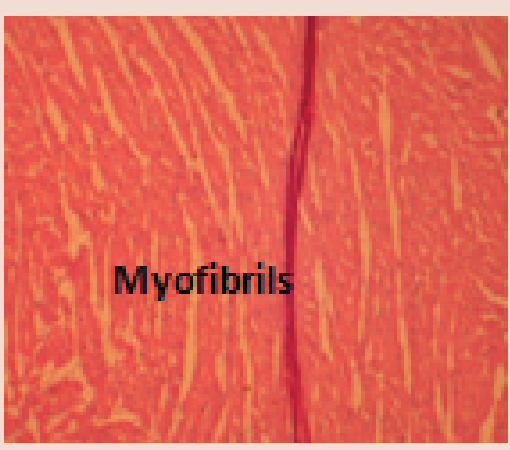

High power photomicrograph of Heart from $\mathrm{HFD}^{\star}$ control group showing edema (E) and change in colour of heart from pink-red to yellowish pink (H\&E x 400).

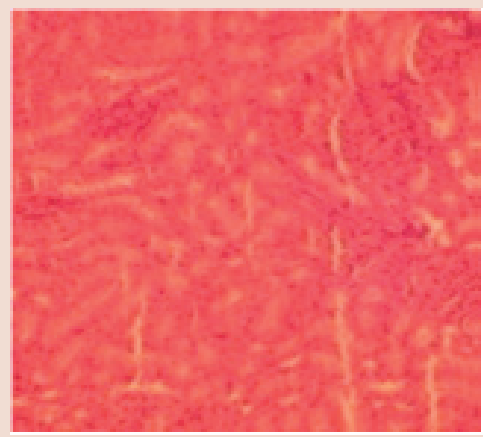

High power photomicrograph of Heart from Z. officinale treated group showing without any significant change with normal arteriole (H\&E x 400).

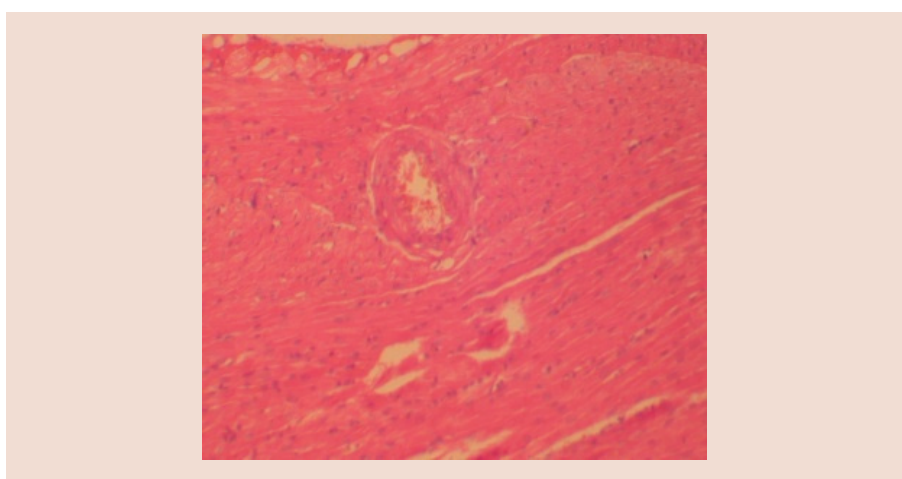

High power photomicrograph of Heart standard group showing normal arteriole (H\&E x 400).

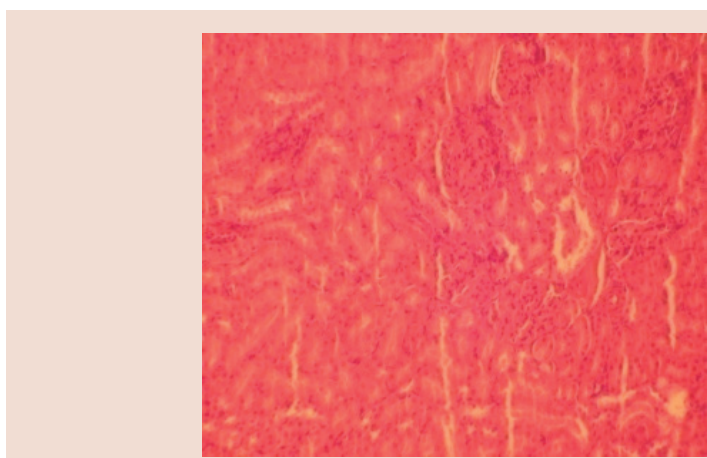

High power photomicrograph of Heart from per se group without any significant change with normal arteriole (H\&E x 400).

Figure 4: Changes observed in heart of different groups

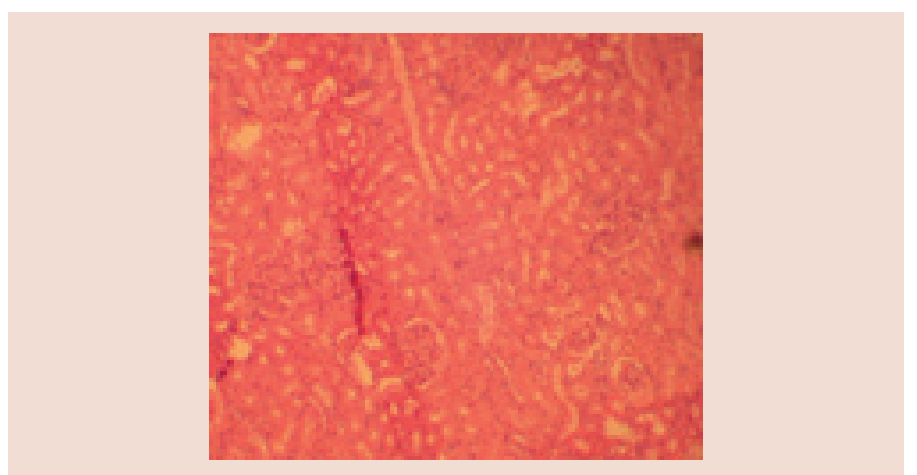

Low power photomicrograph of Kidney from $\mathrm{ND}^{\star}$ control group showing normal glomerulus ( $\mathrm{H} \& \mathrm{E} \times 100)$.

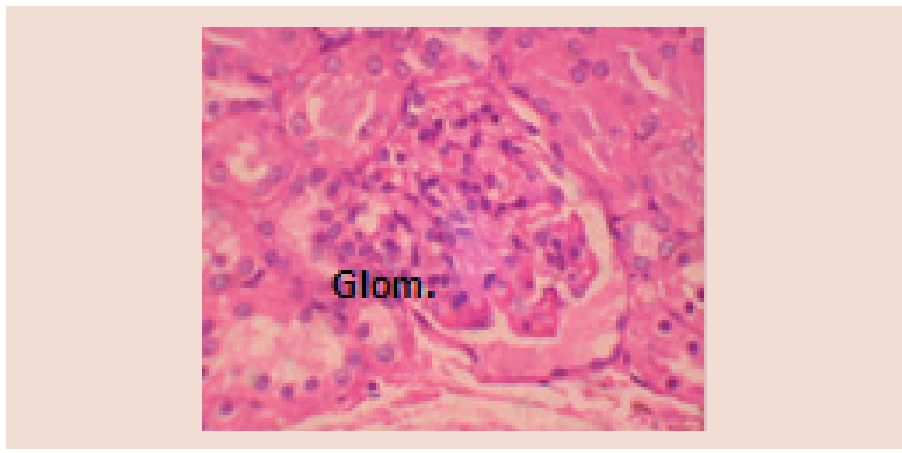

High power photomicrograph of Kidney from $\mathrm{HFD}^{\star}$ control group showing normal glomerulus (G) (H\&E x 400). 


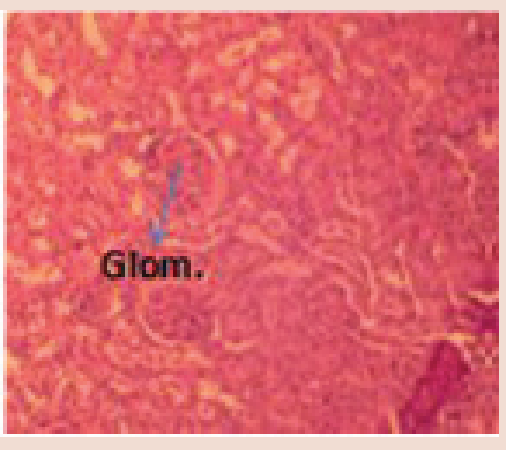

High power photomicrograph of Kidney from Z. officinale treated group showing normal glomerulli (G). (H\&E x 400).

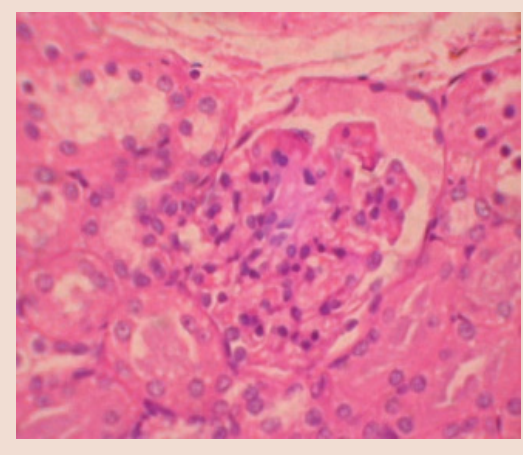

High power photomicrograph of Kidney from standard group showing normal glomerulus (G) (H\&E x 400).

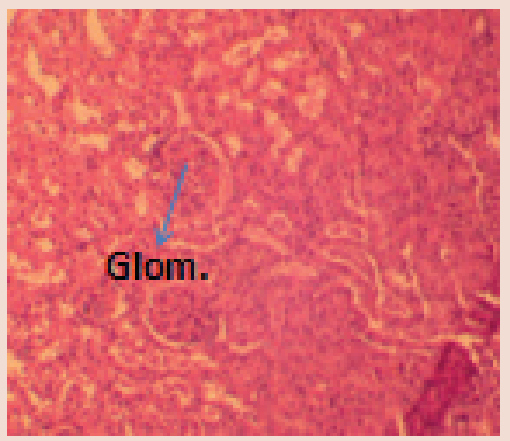

Low power photomicrograph of Kidney from per se group showing normal glomerulli (G) (H\&E x 100).

Figure 5: Changes observed in kidney of different groups

\section{DISCUSSION}

Present study was carried out to investigate the anti-obesity effects of aqueous $Z$. officinale extract in HFD-induced obesity in Wistar rats. Obesity, which affects up to $30 \%$ of the adult population in developed countries. Obesity is associated with serious mortalities including high incidence of type 2 diabetes, hyperlipidemia, hypercholesterolemia, cardiovascular disease and many more. HFD is thought to be one of the main factors for development of obesity. We selected a rat model of diet induced obesity based on numerous experimental studies indicated that consumption of HFD leads to increased body weight and fat mass, induce alterations in carbohydrate and lipid metabolism, lead to insulin resistance, increase production and release of leptin in laboratory animals and humans. ${ }^{10-13}$
In the present study, we found that the body weight was markedly reduced in the animal groups treated by $Z$. officinale to vehicle-treated control. The inhibition of body weight gain did not depend upon a decreased food or energy intake, because there was no significant correlation in diet intake between the control and experimental groups, but was caused by preventing and/or delaying of fat absorption. Similarly, we found that the feeding of $Z$. officinale for 6 weeks suppressed the increases in organ weights of the heart kidney, liver, spleen and adipose tissue weights of mesentric, perirenal and uterine fat pad weights induced by a HFD. On the other hand, it is reported that feeding with a high fat diet in wistar rats leads to increase in weight of body organs such as liver, heart, spleen and both kidneys. It is also reported that high fat diet induces substantial increase in deposition of fat in the mesenteric, perirenal and uterine region in wistar rats.

The present study results showed that compared to that of NC, Serum TG, TC, LDL-C, VLDL-C levels were significantly elevated in HFD fed rats (HFD group) decreased in HFD+ ZO treated group more than that of HFD fed group; indicating potential lipid lowering activity of the test drug.

Among various fat deposits, visceral fat mass correlated to insulin resistance in animal model. ${ }^{14}$ Individuals with visceral by obesity have impaired insulin action, ${ }^{15}$ and prevents insulin resistance by removing visceral fat mass ${ }^{16}$ From adipose tissue, two groups of inflammatory proteins are produced (i) IL- 6 and TNF- $\alpha$ [inflammatory mediators], (ii) leptin, adiponectin and resistin [adipocytokines]. ${ }^{17}$ Human obesity is characterized by variable degrees of hyperleptinemia and leptin resistance. ${ }^{18}$ The result of the present study indicates insulin sensitivity improved in rat fed with the drug. The drug induced melioration of insulin resistance which is supported by down regulation of leptin. Thus, visceral fat accumulation and improved insulin resistance in rat model with HFD induced obesity may possibly be through down regulation of leptin, TNF- $\alpha$, SREBP $1 \gamma$, and PPAR $\gamma 2$ gene expression. Uygun et al reported that leptin might contribute to hepatic steatosis by promoting insulin resistance and also by altering insulin signaling in hepatocytes, which consequently promote increased intracellular fatty acid. ${ }^{19}$ Hence, drug treatment to rats with HFD prevents increase of these levels due to decrease of the body fat content. The $Z$. officinale administration also showed significant activity against lipase activity.

Conclusively, observed reduction in body weight gain, serum lipids, insulin, leptin levels suggests that aqueous extract of $Z$. officinale possess significant anti-obesity potential.

\section{ABBREVIATIONS USED}

ZO: Zingiber officinale; HFD: High Fat Diet; TC: Triglycerides; LDL-C: Low Density Lipoprotein; VLDL-C: Very Low Density Lipoprotein;

HDL-C: High Density Lipoprotein; ORL: Orlistate.

\section{REFERENCES}

1. Srivastava N, Lakhan R, Mittal B. Physiology and genetics of obesity. Ind J of Exp Biol. 2007;45:929-36

2. National Institutes of Health, National Heart, Lung, and Blood Institute, in cooperation with the National Institute of Diabetes and Digestive and Kidney Diseases. Clinical Guidelines on the Identification, Evaluation, and Treatment of Overweight and Obesity in Adults: The Evidence Report. US Government Press, Washington, DC; 1998; NIH Publication No. 98-4083.

3. Bhutani KK, Gohil VM. Natural products drug discovery research in india: status and appraisal. Indian J Exp Biol. 2010;48(3):199-207.

4. Katiyar, SK, Agarwal, R, Mukhtar, H. Inhibition of tumor promotion in SENCAR mouse skin by ethanol extract of Zingiber officinale rhizome. Can Res. 1996;56:1023-30.

5. Kaur, C, Kapoor, HC. Anti-oxidant activity and total phenolic content of some Asian vegetables. Int J of Food Science and Tech. 2002;37(2):153-61.

6. Srivastava KC, MustafaT. Ginger (Zingiber officinale) in rheumatism and muscu- 
loskeletal disorders. Medical hypotheses. 1992;39(4):342-48.

7. Anonymous. Indian Pharmacopoeia. Government of India. New Delhi, India; 1996.

8. Trease GE, Evans WC. Pharmacognosy. Philadelphia: Bailliere Tindal Publisher and distributer. 1989.

9. Friedewald WT, Levy RI, Fredrickson DS. Estimation of the concentration of low density lipoprotein cholesterol in plasma, without use of the preparative ultracentrifuge. Clin Chem. 1972;18(6):499-502.

10. Kaiyala KJ, Prigeon RL, Kahn SE, Woods SC, Porte DJ, Schwartz MW. (1999). Reduced beta-cell function contributes to impaired glucose tolerance in dogs made obese by high fat feeding. Am J Physiol Endocrinol Metab. 1999; 277(4):659-66

11. Kraegen EW, Clark PW, Jenkins AB, Daley EA, Chisholm DJ, Storlien LH. Development of muscle insulin resistance after liver insulin resistance in high-fat-fed rats. Diabetes. 1991;40(11):1397-403.

12. Mooradian AD, Chehade J, Hurd R, Haas MJ. Monosaccharide-enriched diets cause hyperleptinemia without hypophagia. Nutrition. 2000;16(6):439-41.

13. Brown JL, Spicer MT, Spicer LJ. Effect of high-fat diet on body composition and hormone responses to glucose tolerance tests. Endocrine. 2002;19(3): 327-32.

14. Axen K V, Dikeakos A, Sclafani A. High Dietary Fat Promotes Syndrome $X$ in Nonobese rats. J Nutr. 2003;133(7):2244-49.

15. O'Shaughnessy IM, Myeres TJ, Stepniakowski K, Nazzaro P, Kelly TM Hoffmann RG, et al. Glucose metabolism in abdominally obese hypertensive and normotensive subjects. Hypertension. 1995;26(1):186-192.

16. Gabriely I, Ma XH, Atzmon G, Rajala MW, Berg AH, Scherer P, et al. Removal of visceral fat prevents insulin resistance and glucose intolerance of aging: an adipokine-mediated process. Diabetes. 2001;51(10):2951-58.

17. Alam I, Lewis K, Stephenes JW, Baxter JN. Obesity, metabolic syndrome and sleep apnoea: all proinflammatory states. Obes Rev. 2006;8(2):119-27.

18. Friedman JM, Halaas JL. Leptin and the regulation of body weight in mammals Nature. 1998;395(6704):763-70.

19. Uygun A, Kadayifci A, Yesilova Z, Erdil A, Yaman H, Saka M, et al. Serum leptin levels in patients with nonalcoholic steatohepatitis. Am J Gastroenterol. 2000;95(12):3584-89.

\section{SUMMARY}

- On compared to NC, Serum TG, TC, LDLC, VLDL-C levels were significantly elevated in HFD fed rats (HFD group) decreased in HFD+ ZO treated group, indicating potential lipid lowering activity of the test drug.

- Z. officinale extract causes visceral fat accumulation and improved insulin resistance in rat model with HFD induced obesity through down regulation of leptin, TNF- $\alpha$, SREBP1 $\gamma$, and PPAR $\gamma 2$ gene expression.

- Z. officinale administration also showed significant activity against lipase activity by decrease of the body fat content.

- Reduction in body weight gain, serum lipids, insulin, leptin levels suggests that aqueous extract of $Z$. officinale possess significant anti-obesity potential

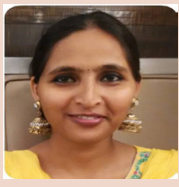

\section{ABOUT AUTHORS}

Iram Nazish: Is a PhD in Pharmacognosy and obtained her doctorate degree from Hamdard University, (New Delhi, India). Her major area of research interest includes herbal drug extraction, standardization of phytochemical compounds and development of novel drug delivery systems of herbal drugs. She has published. She has published more than 20 publications in diverse domains of pharmacognosy and phytochemistry and 1 book chapter, 2 books and 30 monographs to her credit.

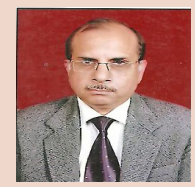

Dr. Ansari: Is serving in Faculty of Pharmacy, Jamia Hamdard (Hamdard University) as professor and Head, Department of Pharmacognosy and Phytochemistry. Hehas also served Jamia Hamdard as Dean, Faculty of Pharmacy and Dean, Students Welfare, Jamia Hamdard. His area of research is quality standards and standardization of herbal formulations of Ayurvedic and Unani drugs. Prof. Ansari has also handled and successfully completed several research projects funded by various government agencies in India.

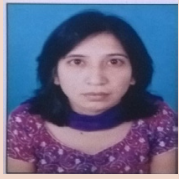

Poonam Arora: Is a Ph.d student, Department of Pharmacognosy and Phytochemistry, Faculty of Pharmacy, Jamia Hamdard(Hamdard University) under guidance of Prof. S. H. Ansari. She has completed her M. Pharm inPharmacognosy and Phytochemistry, with research on investigation of some herbal drugs for their anti-obesity potential. Currently, she is working on quality standards and preclinical studies of Ayurvedicherbal formulation. Miss Poonam has also completed one research project funded by Indian Council of Medical Research (ICMR).

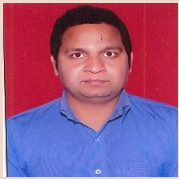

Adil Ahmad: Is a Ph.d student, Department of Pharmacognosy and Phytochemistry, Faculty of Pharmacy, Jamia Hamdard (Hamdard University) under guidance of Prof. S. H. Ansari. He has completed his M. Pharm in Pharmacognosy and Phytochemistry, with research on investigation of some herbal drugs for their Anti-thrombocytopenicpotential in animals. Currently, he is working on standardization, bioactivity guided fractionation and preclinical studies of some herbal drugs in thrombocytopenia. 